\title{
A Comparison of Flat and Shallow Conical Tips for Cervical Cryotherapy
}

\author{
Katbleen A. Stienstra, MD, Benjamin E. Brewer, MD, and LeRoy A. Franklin, PbD
}

Background: Although two types of cervical cryotherapy tips are widely used, there have been no randomized prospective comparison studies reported in the medical literature. Shallow conical tip proponents theorize that a greater depth of freeze near the os yields better treatment of cervical intraepithelial neoplasia (CIN) without elevating the squamocolumnar junction into the cervical canal. Flat tip proponents theorize an equally effective CIN treatment with lower incidence of posttreatment squamocolumnar junction location in the cervical canal.

Methods: A comparative descriptive study was performed to evaluate 117 cryotherapy candidates with biopsy-proved CIN who were classified by location of their squamocolumnar junctions (ectocervix, at the os, or in the canal). They were then randomized to receive double-freeze cervical cryotherapy with either a flat or shallow conical tip. Four or more months later, repeated colposcopy and Papanicolaou smears were performed to assess resolution of $\mathrm{CIN}$ and posttreatment location of the squamocolumnar junction.

Results: Eighty-four patients (71\%) completed the study. Analysis indicated no important difference between the two tips in eliminating CIN. The squamocolumnar junction was colposcopically visualized at all posttreatment examinations. When the pretreatment squamocolumnar junction location was on the ectocervix, data analysis indicated that squamocolumnar junction movement was greater with the shallow conical tip $(P=.037)$, particularly into the canal, where it is clinically more difficult to visualize $(P=.019)$. There were no significant differences in movement of the squamocolumnar junction when it was originally at the os or in the canal.

Conclusions: This study found no significant difference in effectiveness of the two types of tips in eliminating CIN and supports the practice of using one type-either flat or shallow conical tips-to treat all candidates for cervical cryotherapy. Using the flat tip when the pretreatment squamocolumnar junction is on the ectocervix will allow easier posttreatment visualization of the squamocolumnar junction. Further studies with a greater number of subjects are indicated. (J Am Board Fam Pract 1999;12: 360-6.)

Cryotherapy with nitrous oxide using a closed system is an appropriate treatment for cervical intraepithelial neoplasia (CIN) when noninvasive disease has been found on colposcopic examination during which the squamocolumnar junction is visible in its entirety and the limits of the lesion are

Submitted, revised, 31 March 1999.

From the Union Hospital Family Practice Residency (KAS, BEB), and the Department of Statistics, Rose-Hulman-Institute of Technology (LAF), Terre Haute, Ind. Address reprint requests to Kathleen A. Stienstra, MD, Union Hospital Family Practice Residency, 1513 N 61/2 Street, Terre Haute, IN 47807.

This research was partially funded by the American Academy of Family Physicians Foundation. Preliminary results were presented at the Indiana Academy of Family Physicians Annual Research Day, Indianapolis, 1997, and the Biennial Meeting of the Association for Colposcopy and Cervical Pathology, Phoenix, March, 1998. Final results were presented at the Indiana Academy of Family Physicians Annual Research Day, Indianapolis, 1999. completely seen. The directed biopsies should correlate with the Papanicolaou smear, and the findings from an endocervical curettage should be negative. ${ }^{1}$ Lesions greater than $3 \mathrm{~cm}$ in diameter are more successfully treated with laser vaporization $^{2}$ or loop electrosurgical excision procedure (LEEP).

Cryotherapy is initially successful in eliminating cervical dysplasia $76 \%$ to $97 \%$ (average $85 \%$ ) of the time with the first treatment when a double-freeze technique is used, even when the lesion extends up to $5 \mathrm{~mm}$ into the endocervical canal. ${ }^{3-6}$ A second treatment with cryotherapy yields a cure rate of $93 \%$ to $96 \% .^{3,7-10}$ The double-freeze technique is considerably more effective than the single-freeze technique. ${ }^{11}$ Negative findings on a Papanicolaou smear and colposcopy 4 months after cryotherapy accurately predict no recurrences during the ensuing 2 to 3 years. ${ }^{12,13}$ 


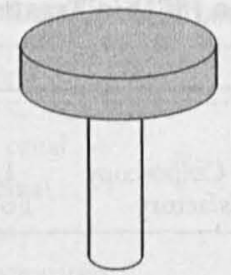

Flat Tip

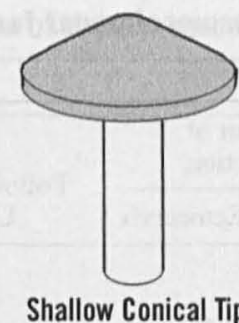

Shallow Conical Tip
Figure 1. Cervical cryotherapy tips.

Cryotherapy is less expensive than laser therapy or LEEP, has fewer complications, and is clinically as effective. ${ }^{2,14-16}$ The major disadvantage of cryotherapy is the resulting highly placed squamocolumnar junction within the cervical canal, thereby making future examinations more difficult and increasing the inadequacy of future colposcopies. $^{2,6,15,16}$ Cryotherapy does not affect subsequent fertility ${ }^{17-19}$ and might actually decrease hostile cervical mucus. ${ }^{20}$ No deleterious effects on labor have been found, although a trend toward more precipitous dilation (defined as less than 3 hours for the first stage of labor) has been reported. ${ }^{21}$

Twenty-four to 48 hours after cervical cryotherapy, the superficial necrotic tissue separates from the stroma because of underlying edema. It is possible to remove the necrotic tissue with gauze and a ring forceps. Study has shown that this practice lessens the posttreatment odor, but not the amount of watery discharge. ${ }^{22,23}$

Currently two types of cryotherapy tips are widely use - a flat tip and a shallow conical tip (Figure 1). These tips are each available in small (19-mm) and large (25-mm) diameters. Choice of tip size is based on the size of the cervical transformation zone and the lesion itself., ${ }^{6,24}$

Advocates of the shallow conical tip contend that it will allow a greater depth of freeze near the os, yielding better treatment of CIN without elevating the squamocolumnar junction too far into the cervical canal. ${ }^{6}$ In the past, tips with a longer nipple configuration were used. Treatment using these tips not only caused the squamocolumnar junction to migrate high into the canal but also caused more cervical stenosis. ${ }^{24,25}$

Advocates of the flat tip contend that CIN treatment is as effective as that obtained with the conical tip, yet the rate at which the posttreatment squamocolumnar junction is located in the cervical canal is reduced. ${ }^{24}$
A literature review indicates a dearth of studies prospectively comparing the efficacy of these two tips in the treatment of CIN or their respective effects on the posttreatment squamocolumnar junction location. Many published studies examining other aspects of cervical cryotherapy do not specify the tip type used, which would allow comparative data analysis. $2,4,7,9,10,14-16,21$

This study was undertaken to test our research hypotheses: (1) shallow conical tips are more effective than flat tips in eliminating CIN; (2) the use of a flat tip leads to fewer future unsatisfactory (squamocolumnar junction not visible) and difficult (squamocolumnar junction in the canal) colposcopic examinations than the shallow conical tip; and (3) the difference in the effect of treatment on the squamocolumnar junction location between a flat tip and a shallow conical tip varies by the pretreatment location of the squamocolumnar junction (on the ectocervix, at the os, or within the cervical canal.)

\section{Methods}

The proposed study was reviewed and approved by the institutional review board of the participating hospital. Study participants were candidates for cervical cryotherapy who had undergone colposcopically directed biopsies in the Family Practice Residency Center (FPC) and had given informed consent. These participants included FPC patients referred internally and patients referred from the local Title $\mathrm{V}$ prenatal clinic, university student health clinic, Planned Parenthood, and other local physicians. A candidate for cryotherapy was not pregnant and had an adequate colposcopic evaluation showing a negative finding on endocervical curettage and CIN that did not extend more than 5 $\mathrm{mm}$ into the canal or involve an area more than 3 $\mathrm{cm}$ in diameter. The criterion of limiting candidates to women whose lesions occupied no more than two quadrants of the cervix was not applied. ${ }^{26}$ The study included women with low-grade and high-grade lesions. Randomization occurred independently of the severity of the lesions. Colposcopic examinations and cryotherapy were performed by one of the investigators (KAS), a family practice resident, or other faculty member under the investigator's supervision.

Patients were first classified into one of three categories based on the location of the squamoco- 
Table 1. Comparison of Cervical Cryotherapy Tips and Location of Squamocolumnar Junction (SCJ) in Treatment of Cervical Intraepithelial Neoplasia (CIN).

\begin{tabular}{|c|c|c|c|c|c|c|c|}
\hline \multirow{2}{*}{$\begin{array}{l}\text { Pretreatment } \\
\text { Anatomy }\end{array}$} & \multirow{2}{*}{$\begin{array}{c}\text { CIN } \\
\text { Resolved }\end{array}$} & \multirow{2}{*}{$\begin{array}{c}\text { CIN } \\
\text { Persistent }\end{array}$} & \multicolumn{3}{|c|}{$\begin{array}{l}\text { Posttreatment Location of } \\
\text { Squamocolumnar Junction }\end{array}$} & \multirow{2}{*}{$\begin{array}{c}\text { Follow-up Colposcopy } \\
\text { Unsatisfactory }\end{array}$} & \multirow{2}{*}{$\begin{array}{l}\text { Lost to } \\
\text { Follow-up }\end{array}$} \\
\hline & & & In Canal & At Os & On Ectocervix & & \\
\hline \multicolumn{8}{|l|}{ Flat tips } \\
\hline SCJ in canal & 7 & 3 & 4 & 6 & 0 & 0 & 3 \\
\hline SCJ at os & 10 & 4 & 4 & 10 & 0 & 0 & 3 \\
\hline SCJ on ectocervix & 14 & 3 & 1 & 13 & 3 & 0 & 7 \\
\hline Total & 31 & 10 & 9 & 29 & 3 & 0 & 13 \\
\hline \multicolumn{8}{|l|}{ Conical tips } \\
\hline SCJ in canal & 7 & 2 & 3 & 6 & 0 & 0 & 3 \\
\hline SCJ at os & 9 & 5 & 3 & 11 & 0 & 0 & 6 \\
\hline SCJ on ectocervix & 17 & 3 & 8 & 12 & 0 & 0 & 11 \\
\hline Total & 33 & 10 & 14 & 29 & 0 & 0 & 20 \\
\hline
\end{tabular}

lumnar junction before treatment: in the canal, at the os, or on the ectocervix. If a Kogan endocervical speculum was required to see the entire squamocolumnar junction, the location was classified as within the canal. The visual differentiation of the other two categories was made by one of the investigators (KAS) who was present at all examinations.

Once stratified to one of these three categories, the patients were then randomly assigned to the flat tip or conical tip treatment group by use of a random number list. This randomization distributed all three squamocolumnar junction types between the two tip types and allowed for subgroup analysis.

A double-freeze technique was used in which freezing took place for a minimum of 3 minutes to achieve a 5- to 7-mm freeze zone and was repeated after a complete thaw. The patient was encouraged, but not required, to return 24 to 36 hours after her cryotherapy to have the necrotic zone of tissue removed. $^{22,23}$

Four or more months after treatment, patients returned for a second Papanicolaou smear and colposcopic examination, which were performed at the same visit. Patient reminders, and if necessary, telephone calls, were used to encourage patients to return. Biopsies were performed only if lesions were visualized on colposcopy. If the Papanicolaou smear showed no dysplasia, and the colposcopic impression or colposcopically guided biopsies indicated no persistent dysplasia, the patient's treatment was considered successful. Patients were then instructed to have further Papanicolaou smears by their personal clinician at 6 and 12 months and annually thereafter.

Posttreatment location of the squamocolumnar junction was classified using the pretreatment categories. If the squamocolumnar junction was not entirely visible, the colposcopic examination was considered unsatisfactory, and cryotherapy was contraindicated should further treatment be indicated.

For statistical analysis of the small subgroups, a test of hypothesis for equality of proportions of resolution for the flat versus shallow conical tip was performed using the Fisher exact test. ${ }^{27}$ An analysis of the effect of tip shape was considered individually for the three possible pretreatment locations of the squamocolumnar junction. An overall test of differences of proportion was undertaken by combining the samples of the three sites. The combined samples were large enough to allow standard normal or $z$-based analysis of proportions. ${ }^{27}$ All tests

Table 2. Analysis of Resolution of Cervical Intraepithelial Neoplasia Using Flat or Conical Tips for Cervical Cryotherapy.

\begin{tabular}{lcccc}
\hline $\begin{array}{l}\text { Squamocolumnar } \\
\text { Junction Location }\end{array}$ & $\begin{array}{c}\text { Flat } \\
\text { No. (\%) }\end{array}$ & $\begin{array}{c}\text { Conical } \\
\text { No. (\%) }\end{array}$ & \multicolumn{2}{c}{$P$ Value } \\
\hline In canal & $7 / 10(70)$ & $7 / 9(78)$ & $.556^{\star}$ & NS \\
At os & $10 / 14(71)$ & $9 / 14(64)$ & $.497^{\star}$ & NS \\
On ectocervix & $14 / 17(82)$ & $17 / 20(85)$ & $.587^{\star}$ & NS \\
Combined groups & $31 / 41(76)$ & $33 / 43(77)$ & $.451^{\dagger}$ & NS \\
\hline
\end{tabular}

*Fisher exact test.

${ }^{t}$ Standard normal or $z$ based. 
Table 3. Analysis of Location of the Squamocolumnar Junction (SCJ) Using a Flat or Conical Cryosurgical Tip.

\begin{tabular}{|c|c|c|c|}
\hline Location & Flat Tip No. (\%) & Conical Tip No. (\%) & $P$ Value \\
\hline SCJ in canal & & & $.570^{*}(\mathrm{NS})$ \\
\hline To canal & $4 / 10(40)$ & $3 / 9(33)$ & \\
\hline To os & $6 / 10(60)$ & $6 / 9(67)$ & \\
\hline To ectocervix & $0 / 10(0)$ & $0 / 9(0)$ & \\
\hline SCJ at os & & & $.500^{*}(\mathrm{NS})$ \\
\hline To canal & $4 / 14(29)$ & $3 / 14(21)$ & \\
\hline To os & $10 / 14(71)$ & $11 / 1+(79)$ & \\
\hline To ectocervix & $0 / 14(0)$ & $0 / 1+(0)$ & \\
\hline SCJ on ectocervix & & & $.037^{\star 1}$ \\
\hline To canal & $1 / 17(6)$ & $8 / 20(40)$ & \\
\hline To os & $13 / 17(76)$ & $12 / 20(60)$ & \\
\hline To ectocervix & $3 / 17(18)$ & $0 / 20(0)$ & \\
\hline No movement vs movement to os canal & 3 vs 14 & 0 vs 20 & $.088^{*}(\mathrm{NS})$ \\
\hline No movement to os vs movement to canal & 16 vs 1 & 12 vs 8 & $.019^{\star \dagger}$ \\
\hline Combined groups & & & $.186^{\ddagger}(\mathrm{NS})$ \\
\hline To canal & $9 / 41(22)$ & $14 / 43(33)$ & \\
\hline To os & $29 / 41(71)$ & $29 / 43(67)$ & \\
\hline To ectocervix & $3 / 41(7)$ & $0 / 43(0)$ & \\
\hline Ectocervix vs os canal & 3 vs 38 & 0 vs 43 & $.112^{*}(\mathrm{NS})$ \\
\hline Ectocervix os vs canal & 32 vs 9 & 29 vs 14 & $.135^{\ddagger}(\mathrm{NS})$ \\
\hline
\end{tabular}

*Fisher exact test.

${ }^{\dagger}$ Significant at the .05 level.

${ }^{\ddagger}$ Standard normal or $z$ based.

were conducted as 1-tailed tests in accordance with the stated research hypotheses.

Data specifying the length of time for follow-up after cryotherapy (4 months or number of months longer) were not collected. Analysis of the characteristics of those lost to follow-up was not performed.

\section{Results}

One hundred seventeen women with biopsyproved cervical dysplasia were randomized to receive cryotherapy with a flat tip or a shallow conical tip. A total of 54 patients received treatment with a flat tip and 63 received treatment with a shallow conical tip. Thirteen patients in the flat tip group were lost to follow-up compared with 20 patients in the conical-tip group. Eighty-four patients (71\%) were evaluated with a follow-up colposcopy. A compilation of the study data is presented in Table 1.

\section{Effectiveness of Conical Versus Flat Tip in CIN Resolution}

The overall documented resolution of CIN was $76 \%$ (64 out of 84 who returned for a follow-up visit at 4 months or more longer). There were no significant differences in proportion of success of resolution of CIN in any category of pretreatment squamocolumnar junction location and no significant difference for the combined samples of three sites. These data are presented in Table 2.

Even when the squamocolumnar junction was in the canal, which is theoretically more difficult to treat, the conical tip resolved 7 of the 9 or $78 \%$ of cases, and the flat tip resolved 7 of the 10 cases or $70.0 \%$.

\section{Effect of Conical and Flat Tip on Unsatisfactory Posttreatment Exams}

All posttreatment colposcopic examinations were satisfactory (squamocolumnar junction was entirely visible.) No difference between the two tip types was found.

\section{Effect of Conical and Flat Tips and Pretreatment Squamocolumnar Junction Location on} Posttreatment Squamocolumnar Junction Location The analysis of the effect of tip shape on squamocolumnar junction movement was done individu- 


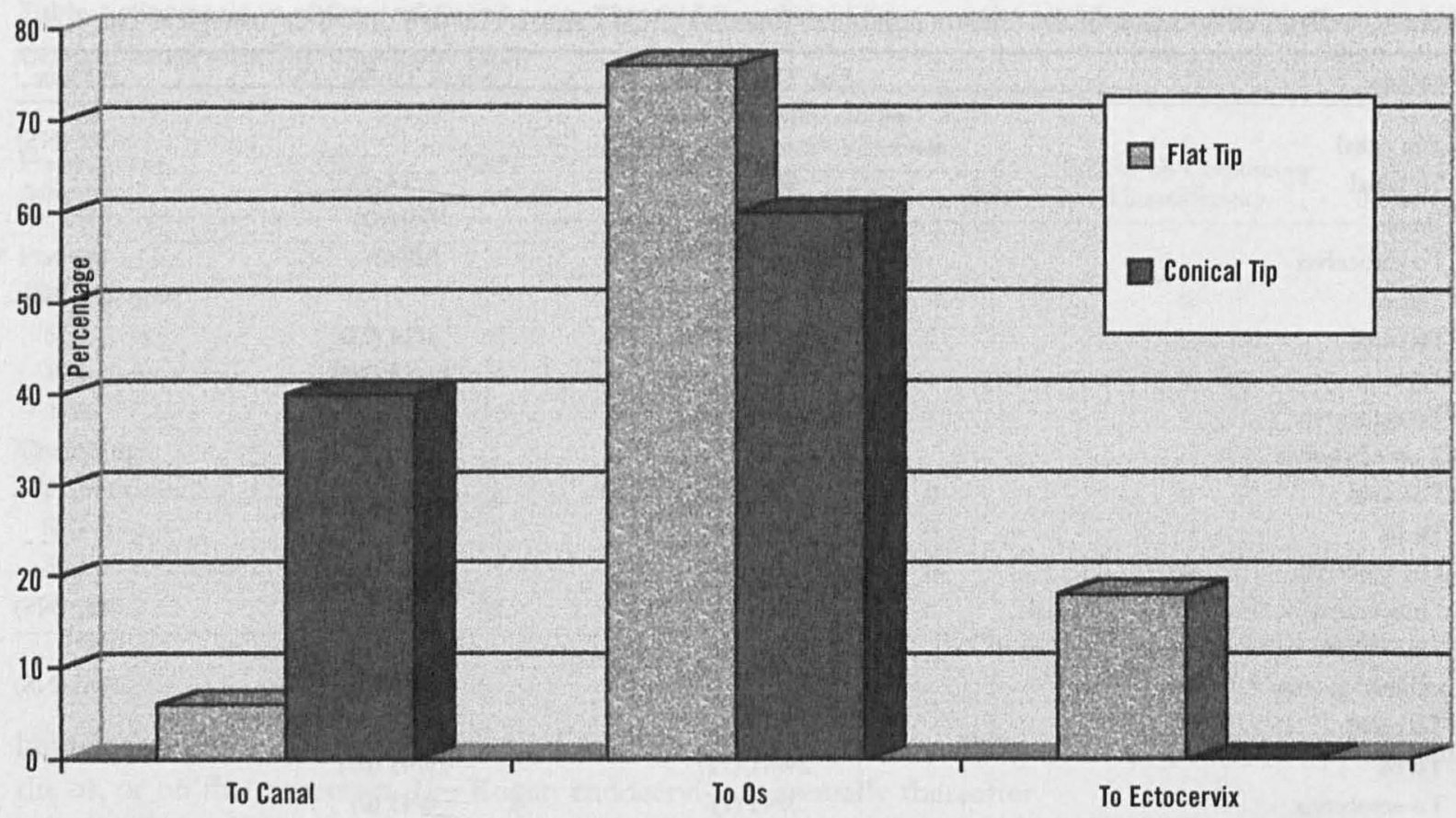

Post-treatment SCJ Location

Figure 2. Analysis of group with pretreatment squamocolumnar junction location on the ectocervix.

ally for the three possible pretreatment locations of squamocolumnar junction. These data are presented in Table 3. No significant difference of the proportion of movement was observed when the squamocolumnar junction was in the canal or was at the os between the two tip shapes.

Figure 2 illustrates the posttreatment squamocolumnar junction location data when the pretreatment squamocolumnar junction was on the ectocervix. A significant difference of proportion of movement was observed for squamocolumnar junction on the ectocervix $(P=.03682)$. This finding indicates that the conical tip results in a higher proportion of shifts of the squamocolumnar junction into the canal after treatment. This analysis compared the groups in which movement of the squamocolumnar junction occurred. The 3 patients that exhibited no shift of the squamocolumnar junction in the flat tip group were not included in this analysis but were in those described next.

Analysis of the group of patients with the squamocolumnar junction on the ectocervix, which compared the two tip types with the "no movement from ectocervix" variable and the "movement from ectocervix to os or into canal" variable, showed no significant difference. A further analysis of this group compared "no movement or movement to the os" with "movement into the canal" (ie, unimportant movement with clinically important movement). This test had a significant difference of proportions $(P=.01856)$. This indicates that when the squamocolumnar junction is initially on the ectocervix, use of the conical tip results in a higher proportion of clinically important movement of the squamocolumnar junction into the canal, where it is more difficult to monitor.

An analysis of the combined samples of the three sites showed no significant difference between the tips for any movement of the posttreatment squamocolumnar junction locations.

\section{Discussion}

Results of this study failed to show a statistically significant difference between the effectiveness of treatment with flat tips and shallow conical tips for eliminating cervical CIN. Location of the squamocolumnar junction before treatment did not influence the effectiveness of treatment. Our data do not support the assumption that the shallow conical tip provides a better freeze zone into the canal. 
Neither the flat tip nor the shallow conical tip caused movement of the squamocolumnar junction far enough to limit future colposcopic examinations. There were no unsatisfactory follow-up colposcopic examinations in either group.

The results of this study suggest that the flat tip is preferable for patients with a squamocolumnar junction on the ectocervix because it is as effective as the shallow conical tip in resolving CIN and it facilitates an easier follow-up examination. When the squamocolumnar junction was initially on the ectocervix, the conical tip resulted in movement of the squamocolumnar junction into the cervical canal significantly more often, where it is more difficult to monitor. A squamocolumnar junction in the canal requires the use of an endocervical speculum for complete visualization-a technically more difficult procedure.

For the groups whose pretreatment squamocolumnar junctions were located at the os or in the canal, our data did not show any statistically significant difference between using the flat and shallow conical tips in the posttreatment squamocolumnar junction location.

Our study documented a success rate of $76 \%$ in eliminating CIN. This rate is in the low range of the $76 \%$ to $97 \%$ (average $85 \%$ ) primary cure rate reported in the literature. ${ }^{3,4,6,25}$ A possible explanation for this low rate includes use of the widely applied minimum of 3-minute freezes with a visually estimated freeze zone of 5 to $7 \mathrm{~mm}$. The work of Boonstra et $\mathrm{al}^{28}$ indicates that two freezes of 5 minutes each achieve a much more consistent frozen lesion of the necessary depth, especially at the 3 and 9 o'clock locations. It is unknown whether a longer freeze with the two different tips would affect the posttreatment location of the squamocolumnar junction differently from that found in this study. A device that more accurately measures the freeze zone, rather than a visual estimate, might have led to more effective freezes. ${ }^{29}$ The success rate might have been higher had we applied the criterion of treating only women with lesions confined to two quadrants of the cervix, as some advocate. $^{25,26}$

The patients lost to follow-up rate of $28 \%$ is consistent with that reported in the literature. Pfenninger reported a rate of $40 \%$ lost to follow-up for the first 200 cases of cervical cryotherapy in a Michigan family practice residency setting. ${ }^{30}$ It is unknown whether those patients who did not re- turn for a follow-up visit differed in characteristics from those who returned for further evaluation.

What is the clinical relevance of this study for the physician who uses cryotherapy to treat CIN? Both types of tips are successful in eliminating CIN, and no unsatisfactory posttreatment examinations were found. Both types of tips can be used with confidence and without fear of increasing future conizations. Cryotherapy remains an option that is attractive and more cost-effective than LEEP for the appropriate candidate with CIN. When the pretreatment squamocolumnar junction is on the ectocervix, using the flat tip will allow an easier posttreatment visualization of the squamocolumnar junction.

Power analysis was conducted for our sample size of 41 for the overall resolution of CIN, assuming a resolution rate of $85 \%$ for the conical tip and $75 \%$ for the flat tip. Our power was 0.367 . In other words, there is a $37 \%$ likelihood that similar studies will find a statistically significant difference if there is a $10 \%$ difference in efficacy between the two tips. Additional power analysis of the posttreatment squamocolumnar junction location was performed using our sample values of $22 \%$ migration into the canal for the flat tip and $33 \%$ for the shallow conical tip. Our sample size of 41 would only have a power of 0.267 if there were an $11 \%$ difference. Accordingly, approximately one of four studies similar to ours would have found a statistically significant difference.

Limitations of this study included small sample size and an unblinded primary investigator. More study patients are needed to find further statistically significant differences between the two tip types. It is unknown whether the population of women tested in this study reflects the general population. Factors that were not evaluated with this study and that could be considered with a larger study include characteristics of women who did not return for follow-up, severity of CIN distribution among the groups, smoking status, length of time until follow-up, and characteristics and outcome of women who returned for debridement compared with those who did not. Data suggest that, compared with LEEP, cryotherapy improves resolution of human papilloma virus (HPV) infection because it leaves inactivated viral DNA for antigen presentation. The inactivated DNA boosts local immunity and enhances the woman's return to HPV-negative status. ${ }^{31}$ It is possible that remov- 
ing the eschar after cryotherapy could affect outcome.

Further study is indicated and encouraged.

\section{References}

1. Figge DC, Creasman WT. Cryotherapy in the treatment of cervical intraepithelial neoplasia. Obstet Gynecol 1983;62:353-8.

2. Ferenczy A. Comparison of cryo- and carbon dioxide laser therapy for cervical intraepithelial neoplasia. Obstet Gynecol 1985;66:793-8.

3. Berget A, Lenstrup C. Cervical intraepithelial neoplasia. Examination, treatment, and follow-up. Obstet Gynecol Surv 1985;40:545-52.

4. Bryson SC, Lenehan P, Lickrish GM. The treatment of grade 3 cervical intraepithelial neoplasia with cryotherapy: an 11 year experience. Am J Obstet Gynecol 1985;151:201-6.

5. Krebs HB, Wheelock JB. Endocervical curettage after cryotherapy for cervical intraepithelial neoplasia. J Reprod Med 1985;30:379-82.

6. Campion M, Ferris D, Di Paola F, Reid R. Modern colposcopy: a practical approach. Hagerstown, $\mathrm{Md}$ : Educational Systems, 1991:11-2.

7. Coney P, Walton LA, Edelman DA, Fowler WC Jr. Cryosurgical treatment of early cervical intraepithelial neoplasia. Obstet Gynecol 1983;62:463-6.

8. Creasman WT, Hinshaw WM, Clarke-Pearson DL. Cryosurgery in the management of cervical intraepithelial neoplasia. Obstet Gynecol 1984;63:145-9.

9. Kirwan PH, Smith IR, Naftalin NJ. A study of cryosurgery and the $\mathrm{CO}_{2}$ laser in treatment of carcinoma in situ (CIN III) of the uterine cervix. Gynecol Oncol 1985;22:195-200.

10. van Lent $M$, Trimbos JB, Heintz AP, van Hall EV. Cryosurgical treatment of cervical intraepithelial neoplasia (CIN III) in 102 patients. Gynecol Oncol $1983 ; 16: 240-5$.

11. Schantz A, Thormann L. Cryosurgery for dysplasia of the uterine ectocervix. A randomized study for the efficacy of the single- and double-freeze techniques. Acta Obstet Gynecol Scand 1984;63:417-20.

12. Falcone T, Ferenczy A. Cervical intraepithelial neoplasia and condyloma: an analysis of diagnostic accuracy of posttreatment follow-up methods. Am J Obstet Gynecol 1986;154:260-4.

13. Levine RU, Carillo EJ, Crum CP. Outpatient management of cervical intraepithelial neoplasia. A summary. J Reprod Med 1985;30:351-4.

14. Mitchell MF, Tortolero-Luna G, Cook E, Whittaker L, Rhodes-Morris H, Silva E. A randomized clinical trial of cryotherapy, laser vaporization, and loop electrosurgical excision for treatment of squamous intraepithelial lesions of the cervix. Obstet Gynecol 1998;92:737-44.

15. Kwikkel HJ, Helmerhorst TJ, Bezemer PD, Quaak
MJ, Stolk JG. Laser or cryotherapy for cervical intraepithelial neoplasia: a randomized study to compare efficacy and side effects. Gynecol Oncol 1985; 22:23-31.

16. Wetchler SJ. Treatment of cervical intraepithelial neoplasia with the $\mathrm{CO}_{2}$ laser: laser versus cryotherapy. A review of effectiveness and cost. Obstet Gynecol Surv 1984;39:469-73.

17. Weed JC Jr, Curry SL, Duncan ID, Parker RT, Creasman WT. Fertility after cryosurgery of the cervix. Obstet Gynecol 1978;52:245-6.

18. Montz FJ. Impact of therapy for cervical intraepithelial neoplasia on fertility. Am J Obstet Gynecol 1996;175(4 Pt 2):1129-36.

19. Spitzer M. Fertility and pregnancy outcome after treatment for cervical intraepithelial neoplasia. J Lower Genital Tract Dis 1998;2:225-30.

20. Baram A, Paz GF, Peyser MR, Schachter A, Homonnai ZT. Treatment of cervical ectropion by cryosurgery: effect on cervical mucus characteristics. Fertil Steril 1985;43:86-9.

21. Benrubi GI, Young $M$, Nuss RC. Intrapartum outcome of term pregnancy after cervical cryotherapy. $\mathrm{J}$ Reprod Med 1984;29:251-4.

22. Harper D, Ferris D, Mayeaux E, et al. Hydrorrhea from cryosurgery is not diminished by cervical debridement. Paper presented at the Ninth World Congress, Cervical Pathology and Colposcopy, Sidney, Australia, May, 1996.

23. Nahhas WA, Whitney CW, Rine J. Evaluation of removing devitalized cervical tissue in the reduction of vaginal discharge after cervical cryotherapy: a prospective study. J Reprod Med 1981;26:263-4.

24. Pfenninger J, Fowler GC. Procedures for primary care physicians. St Louis: Mosby-Year Book, 1994: 645-52.

25. Mayeaux EJ Jr, Spigener SD, German JA. cryotherapy of the uterine cervix. J Fam Pract 1998;47:99102.

26. Ferris D. Cryotherapy of the cervix. J Lower Genital Tract Dis 1998;2:98-105.

27. Rosner B, Fundamentals of biostatistics, 2nd ed. Boston: P W S Publishers, 1986:322.

28. Boonstra H, Koudstaal J, Oosterhuis JW, et al. Analysis of cryolesions in the uterine cervix: application techniques, extension, and failures. Obstet Gynecol 1990;75:232-9.

29. Ferris DG, Crawley GR, Baxley E, et al. Cryotherapy precision:. Clinician's estimate of cryosurgical iceball lateral spread of freeze. Arch Fam Med 1993; 2:269-75.

30. Pfenninger JL. Colposcopy in a family practice residency: the first 200 cases. J Fam Pract 1992;34:6772.

31. Nuovo GJ, Pedemonte BM. Human papillomavirus types and recurrent cervical warts. JAMA 1990;263: $1223-6$. 